\title{
Harmonic Analysis of Lossy, Piezoelectric Composite Transducers using the Plane Wave Expansion Method
}

\author{
Leigh-Ann Orr $^{\dagger}$, Anthony J. Mulholland ${ }^{\dagger *}$, Richard L. O’Leary ${ }^{\ddagger}$ \\ and Gordon Hayward ${ }^{\ddagger}$ \\ $\dagger$ Department of Mathematics, University of Strathclyde, \\ Glasgow, UK G1 1XH \\ $\ddagger$ Department of Electronic and Electrical Engineering, \\ University of Strathclyde, Glasgow, UK G1 1XW
}

December 6, 2006

*Department of Mathematics, University of Strathclyde, Livingstone Tower, 26 Richmond Street, Glasgow G1 1XH, U.K., Tel: ++44 (0)141 548 2971, Fax: ++44 (0)141 548 3345, email: ajm@maths.strath.ac.uk 


\begin{abstract}
Periodic composite ultrasonic transducers offer many advantages but the periodic pillar architecture can give rise to unwanted modes of vibration which interfere with the piston like motion of the fundamental thickness mode. In this paper, viscoelastic loss is incorporated into a three dimensional plane wave expansion model (PWE) of these transducers. A comparison with experimental and finite element data is conducted and a design to damp out these lateral modes is investigated. Scaling and regularisation techniques are introduced to the PWE method to reduce ill-conditioning in the large matrices which can arise. The identification of the modes of vibration is aided by examining profiles of the displacements, electrical potential and Poynting vector. The dispersive behaviour of a 2-2 composite transducer with high shear attenuation in the passive phase is examined. The model shows that the use of a high shear attenuation filler material improves the frequency band gap surrounding the fundamental thickness mode.
\end{abstract}

Keywords: COMPOSITES, ULTRASONIC TRANSDUCER, PLANE WAVE EXPANSION, VISCOELASTIC LOSS

PACS Code: $43.35 .+\mathrm{d}$ 


\section{Introduction}

Periodic composite transducers are generally accepted as the design of choice in many biomedical [1], sonar [2] and nondestructive testing applications [3]. This is due to the constituent materials combining to realise better operational characteristics, coupled with the availability of new materials $[4,5]$. The most frequently used designs are manufactured by dicing the ceramic into a series of pillars and then filling the void with a passive polymer phase [6]. The 1-3 design has connectivity in only one direction for the ceramic phase but in all three directions for the polymer phase. For the 2-2 design, the ceramic is cut longitudinally in one direction so that there is connectivity in two directions for both the ceramic and polymer phases. However, one of the problems with this architecture is the presence of parasitic waves, which are generated between adjacent pillars (interpillar modes) or within the pillars (intra-pillar modes), interfering with the piston like behaviour of the fundamental thickness mode [7]. Extensive experimental observations have highlighted the intricate dependency between the geometry of the design, the material properties and the key operational characteristics of the device. It has been suggested that a passive material with a low transverse coupling would enhance the transducer's efficiency $[7,8]$.

There have been numerous theoretical approaches to modelling non-piezoelectric (electrically passive) composite materials. Shui et al studied striated passive composite materials and, by neglecting loss mechanisms, formulated a mechanical model in each phase and applied a set of interface conditions [9]. This partial wave expansion method facilitated the study of the effects of varying the volume fraction of each constituent on the lateral resonant frequencies. These typically provide the upper and lower limits of any stop band gap which encompasses the main mode of vibration. Geng and Zhang used a similar approach for passive materials and found that if the thickness resonant frequency is lower than the lateral mode, then there will be a frequency near the ceramic thickness mode where both constituents vibrate in phase [10]. Certon et al studied the lateral modes of an infinitesimally thin (membrane model) passive composite [11]. This simplified the previous model but restricts the analysis to stop band gaps in the trans- 
verse modes and, by its very nature, does not permit variations in material parameters in the thickness mode direction. It was found that a large ceramic width to pitch ratio resulted in the largest frequency band gaps around the thickness mode. An alternative approach is to capture the spatial variations in the material properties using a Fourier series expansion. Vasseur et al used this plane wave expansion (PWE) method to study the modal behaviour of an anisotropic passive material consisting of a regular array of uni-directional cylindrical fibres embedded in an epoxy matrix [12]. They found that a marked contrast between the two constituent material parameters provides the best opportunities for realising large band gaps. The membrane model was also studied using this method [13] and this highlighted the flexibility of the approach in studying irregular geometries.

There has also been some theoretical investigation of piezoelectric (electrically active) composite transducers using finite element (FE) modelling [14, 15]. Such models can incorporate realistic operating conditions, such as viscoelastic loss, backing and matching layers, mechanical and electrical loads and electrode patterning. The major drawback is the high computational cost. FE modelling can be used in the frequency domain [16] but for the modelling of periodic composite transducers the method is often used in the time domain [19]. In the time domain approach the harmonic analysis requires a numerical Fourier transform of the predicted displacements, which can be problematic if the mode amplitudes have a large variance. Due to the computational cost, a quarter symmetry of a unit cell with appropriate periodic boundary conditions is often employed. For regular geometries this does not present a problem but essentially excludes the study of irregular designs in three dimensions. FE modelling has recently shown that Lamb wave propagation is responsible for inter-element crosstalk and has a detrimental effect on beam forming and transducer sensitivity [4]. It has been suggested that a passive filler with high shear loss may aid the damping of these unwanted Lamb waves [17].

In this paper viscoelastic loss is incorporated into the 3-dimensional PWE model [18]. Loss is vital for reducing crosstalk between adjacent ceramic pillars in the transducer, it facilitates a comparison with experimental results and is necessary for any discussion 
regarding the use of alternative polymer phases. Scaling and regularisation techniques are introduced to reduce ill-conditioning in the large matrices which arise in this method. The identification of the modes is aided by examining profiles of the displacements and the Poynting vector. As mentioned previously, FE modelling $[4,5]$ can lose important information on low amplitude modes and high frequency modes but since the PWE method operates in the frequency domain no such restrictions apply.

In the next section the geometry of the transducer is described in terms of a Fourier series and then the PWE method and associated boundary conditions are briefly outlined. It follows the derivation of Wilm et al [18], with an alternative notation for the Fourier coefficient indexing. As such only a brief outline is given to clarify the later discussions on the inclusion of viscoelastic loss and numerical implementation issues. In Section 3 the inclusion of viscoelastic loss into the model is described, followed by a discussion on the use of scaling and regularisation in the method's implementation. In Section 4 a comparison between the PWE method, FE modelling and experimentally measured behaviour is reported. Finally, a composite transducer with a high shear loss passive phase is examined. Dispersion curves, electrical impedance plots and a modal analysis using displacement and Poynting vector profiles are used to discuss the operating characteristics.

\section{Formulation of the method}

\subsection{The geometry}

The model is configured for periodic 2-2 and 1-3 composites with thickness in the $x_{3}$ direction (see Figure 1). By using the periodicity of the structure the material constants, $M(r)$, can be expanded as Fourier series where $M$ represents either the density $\rho$, the elasticity tensor $c_{i j k l}$, the piezoelectric stress tensor $e_{i j k}$, or the permittivity tensor $\epsilon_{i j}$.

Figure 1:

For the 1-3 composite structure shown in Figure 1 the material constants only depend on $x_{1}$ and $x_{2}$. Denote by $p_{1}$ the period of the geometry in the $x_{1}$ direction and by $p_{2}$ the 
period of the geometry in the $x_{2}$ direction. The width of the ceramic pillar in the $x_{1}$ and $x_{2}$ direction is denoted by $s$ and $t$, respectively. A Fourier series representation for this geometry can be written

$$
M\left(x_{1}, x_{2}\right)=\sum_{m=-\infty}^{\infty} \sum_{n=-\infty}^{\infty} M_{m n} e^{-\jmath\left(\frac{2 \pi m}{p_{1}} x_{1}+\frac{2 \pi n}{p_{2}} x_{2}\right)}
$$

where

$$
M_{m n}=\frac{(\phi-\theta)}{\pi^{2} m n} \sin \left(\frac{\pi m s}{p_{1}}\right) \sin \left(\frac{\pi n t}{p_{2}}\right), \quad(m, n \neq 0)
$$

$M_{00}=\theta+(\phi-\theta) s t / p_{1} p_{2}, M_{m 0}=(\phi-\theta) t /\left(p_{2} \pi m\right) \sin \left(\pi m s / p_{1}\right), M_{0 n}=(\phi-\theta) s /\left(p_{1} \pi n\right) \sin \left(\pi n t / p_{2}\right)$ and $\phi$ and $\theta$ are the particular material property in the ceramic phase and polymer phase respectively. This double subscript notation can be simplified by ordering the Fourier coefficients. Define the ordered set

$$
H=\{(-N,-M),(-N,-M+1), \ldots,(-N, M),(-N+1,-M), \ldots,(N, M)\}
$$

so that if

$$
G^{s}=\left(\frac{2 \pi}{p_{1}} H^{s, 1}, \frac{2 \pi}{p_{2}} H^{s, 2}, 0\right),
$$

then (1) can be rewritten for a finite number of terms $\left(N\right.$ in direction $x_{1}$ and $M$ in direction $x_{2}$ ) as

$$
M\left(x_{1}, x_{2}\right)=\sum_{s=1}^{(2 N+1)(2 M+1)} M^{s} e^{-\jmath G^{s} \cdot r}
$$

where $H^{s, i}$ is the $i^{\text {th }}$ component of element $s$ of $H$. The dependent variables $F(r, t)$ propagating within these periodic structures are approximated as Floquet series

$$
F(r, t, k, \omega)=\sum_{s=1}^{(2 N+1)(2 M+1)} F^{s}(k, \omega) e^{\jmath\left(\omega t-k \cdot r-G^{s} \cdot r\right)}
$$

where $r=\left(x_{1}, x_{2}, x_{3}\right), t$ is time, $\omega$ is the angular frequency and $k=\left(k_{1}, k_{2}, k_{3}\right)$ is the wave vector. 


\subsection{The Model}

The piezoelectric constitutive equations, together with Newton's second law and Gauss's law for dielectric media are [20]

$$
\begin{aligned}
T_{i j} & =c_{i j k l} u_{k, l}+e_{l i j} \phi_{, l} \\
D_{i} & =e_{i k l} u_{k, l}-\epsilon_{i l} \phi_{, l} \\
\rho \frac{\partial^{2} u_{j}}{\partial t^{2}} & =T_{i j, i} \\
D_{i, i} & =0 .
\end{aligned}
$$

Equations (7) to (10) constitute 16 equations in the 16 unknowns which are the stresses $T_{i j}$, the displacements $u_{k}$, the electric potential $\phi$ and the electrical displacements $D_{i}$. Denote the generalized displacement field by $u$ where $u=\left(u_{1}, u_{2}, u_{3}, u_{4}=\phi\right)$ and the generalized stress vectors by $t_{i}=\left(T_{i 1}, T_{i 2}, T_{i 3}, D_{i}\right)$. Substituting the expansion (6) into (7) and (8), and equating coefficients, gives

$$
T_{i j}^{p}=\sum_{q=1}^{(2 N+1)(2 M+1)}-\jmath\left(k_{l}+G_{l}^{q}\right)\left(c_{i j k l}^{V^{p, q}} u_{k}^{q}+e_{l i j}^{V^{p, q}} u_{4}^{q}\right)
$$

and

$$
D_{i}^{p}=\sum_{q=1}^{(2 N+1)(2 M+1)}-\jmath\left(k_{l}+G_{l}^{q}\right)\left(e_{i k l}^{V^{p, q}} u_{k}^{q}-\epsilon_{i l}^{V^{p, q}} u_{4}^{q}\right)
$$

where the particular Floquet series component is given by

$$
V^{p, q}= \begin{cases}p+\frac{(2 N+1)(2 M+1)+1}{2}-q, & \text { if } 1 \leq p+\frac{(2 N+1)(2 M+1)+1}{2}-q \leq(2 N+1)(2 M+1) \\ & \text { and }\left|H^{p, 1}-H^{q, 1}\right| \leq N\left|H^{p, 2}-H^{q, 2}\right| \leq M \\ 0, & \text { otherwise }\end{cases}
$$


and $M^{V^{p, q}}=0$ if $V^{p, q}=0$. In terms of the generalized stress vectors (11) and (12) give

$$
\jmath_{i}^{p}=\sum_{q=1}^{(2 N+1)(2 M+1)}\left(k_{l}+G_{l}^{q}\right) A_{i l}^{p, q} u^{q}
$$

where

$$
A_{i l}^{p, q}=\left[\begin{array}{cccc}
c_{i 11 l}^{V^{p, q}} & c_{i 12 l}^{V^{p, q}} & c_{i 13 l}^{V^{p, q}} & e_{l i 1}^{V^{p, q}} \\
& & & \\
c_{i 21 l}^{V^{p, q}} & c_{i 22 l}^{V^{p, q}} & c_{i 23 l}^{V^{p, q}} & e_{l i 2}^{V^{p, q}} \\
& & & \\
c_{i 31 l}^{V^{p, q}} & c_{i 32 l}^{V^{p, q}} & c_{i 33 l}^{V^{p, q}} & e_{l i 3}^{V^{p, q}} \\
& & & \\
e_{i 1 l}^{V^{p, q}} & e_{i 2 l}^{V^{p, q}} & e_{i 3 l}^{V^{p, q}} & -\epsilon_{i l}^{V^{p, q}} \\
& & &
\end{array}\right] .
$$

The same analysis can be carried out for equations (9) and (10) to obtain the expression

$$
\left(k_{i}+G_{i}^{p}\right) j t_{i}^{p}=\sum_{q=1}^{(2 N+1)(2 M+1)} \omega^{2} R^{p, q} u^{q}
$$

where

$$
R^{p, q}=\left[\begin{array}{cccc}
\rho^{V^{p, q}} & 0 & 0 & 0 \\
0 & \rho^{V^{p, q}} & 0 & 0 \\
0 & 0 & \rho^{V^{p, q}} & 0 \\
0 & 0 & 0 & 0
\end{array}\right]
$$

Now let $T_{i}=\left[t_{i}^{1}, \ldots, t_{i}^{p}, \ldots, t_{i}^{(2 N+1)(2 M+1)}\right]^{T}, U=\left[u^{1}, \ldots, u^{q}, \ldots, u^{(2 N+1)(2 M+1)}\right]^{T}$,

$$
A_{i j}=\left[\begin{array}{cclc}
A_{i j}^{1,1} & A_{i j}^{1,2} & \ldots & A_{i j}^{1,(2 N+1)(2 M+1)} \\
A_{i j}^{2,1} & A_{i j}^{2,2} & \ldots & A_{i j}^{2,(2 N+1)(2 M+1)} \\
\vdots & \vdots & \ddots & \vdots \\
A_{i j}^{(2 N+1)(2 M+1), 1} & A_{i j}^{(2 N+1)(2 M+1), 2} & \ldots & A_{i j}^{(2 N+1)(2 M+1),(2 N+1)(2 M+1)}
\end{array}\right] \text {, }
$$


and

$$
R=\left[\begin{array}{cccc}
R^{1,1} & R^{1,2} & \ldots & R^{1,(2 N+1)(2 M+1)} \\
R^{2,1} & R^{2,2} & \ldots & R^{2,(2 N+1)(2 M+1)} \\
\vdots & \vdots & \ddots & \vdots \\
R^{(2 N+1)(2 M+1), 1} & R^{(2 N+1)(2 M+1), 2} & \ldots & R^{(2 N+1)(2 M+1),(2 N+1)(2 M+1)}
\end{array}\right] .
$$

Equations (14) and (16) can then be written compactly as

$$
\jmath T_{i}=A_{i j} \Gamma_{j} U
$$

and

$$
\omega^{2} R U=\Gamma_{i}\left(\jmath T_{i}\right)
$$

where

$$
\Gamma_{i}=\left[\begin{array}{cccc}
\left(k_{i}+G_{i}^{1}\right) I_{4} & 0 & \ldots & 0 \\
0 & \left(k_{i}+G_{i}^{2}\right) I_{4} & \ldots & 0 \\
\vdots & \vdots & \ddots & \vdots \\
0 & 0 & \ldots & \left(k_{i}+G_{i}^{(2 N+1)(2 M+1)}\right) I_{4}
\end{array}\right] .
$$

Equations (20) and (21) can be combined to give the generalised eigenvalue problem

$$
\left[\begin{array}{cc}
\omega^{2} R-B & 0 \\
-C_{2} & I
\end{array}\right]\left(\begin{array}{c}
U \\
j T_{3}
\end{array}\right)=k_{3}\left[\begin{array}{cc}
C_{1} & I \\
D & 0
\end{array}\right]\left(\begin{array}{c}
U \\
j T_{3}
\end{array}\right)
$$

in the $8(2 N+1)(2 M+1)$ eigenvalues $k_{3}^{(r)}$ and corresponding eigenvectors $\left[\begin{array}{c}U \\ J T_{3}\end{array}\right]^{(r)}$ where $B=\sum_{i, j=1,2} \Gamma_{i} A_{i j} \Gamma_{j}, C_{1}=\sum_{i=1,2} \Gamma_{i} A_{i 3}, C_{2}=\sum_{j=1,2} A_{3 j} \Gamma_{j}$ and $D=A_{33}$. Solving equation (22) and introducing the relative amplitudes $A^{(r)}$ gives

$$
\left(\begin{array}{l}
u(r, t) \\
t_{3}^{q}(r, t)
\end{array}\right)=e^{\jmath\left(\omega t-k_{1} x_{1}-k_{2} x_{2}\right)} \sum_{q=1}^{(2 N+1)(2 M+1)} e^{-\jmath G^{q} \cdot r}\left(\sum_{r=1}^{8(2 N+1)(2 M+1)} A^{(r)} e^{-\jmath k_{3}^{(r)} x_{3}}\left[\begin{array}{l}
u^{q} \\
t_{3}^{q}
\end{array}\right]^{(r)}\right)
$$

Energy distribution within the transducer can be used to clarify particular types of modes in conjunction with examining profiles of the displacements, stresses and electric 
potential. The energy distribution in the device can be examined using the Poynting vector, defined as

$$
P_{j}=-T_{i j} u_{i, t}+\phi D_{j, t}
$$

Substituting equations (7) and (8) into equation (24) gives

$$
P_{j}=-\jmath \omega\left(c_{i j k l} u_{k, l}+e_{l i j} \phi_{, l}\right) u_{i}+\jmath \omega \phi\left(e_{j k l} u_{k, l}-\epsilon_{j l} \phi_{, l}\right)
$$

\subsection{Boundary conditions}

The method is sufficiently general to cope with a wide range of boundary conditions but for simplicity the mechanical boundary conditions of a stress free plate are considered. From equation (23)

$$
0=\sum_{r=1}^{8(2 N+1)(2 M+1)} A^{(r)} e^{-\jmath k_{3}^{(r)} h}\left(T_{3 i}^{q}\right)^{(r)}, \quad q=1, \ldots,(2 N+1)(2 M+1)
$$

and

$$
0=\sum_{r=1}^{8(2 N+1)(2 M+1)} A^{(r)}\left(T_{3 i}^{q}\right)^{(r)}, \quad q=1, \ldots,(2 N+1)(2 M+1) .
$$

For the electrical boundary conditions the electrical potentials at the top and bottom of the transducer are prescribed. The lower surface is a monolithic plate with zero electrical potential. The upper plate has a set of electrodes which follow a periodic spatial pattern. The top surface electrical potential is therefore described by

$$
\phi\left(x_{1}, x_{2}, t\right)=V_{0} e^{\jmath\left(\omega t-\gamma_{1} x_{1}-\gamma_{2} x_{2}\right)},
$$

where $\gamma_{i}=k_{i} p_{i} /(2 \pi)(i=1,2)$ denotes the electrode spacing. This has been nondimensionalised as the ratio of the periodicity of the device architecture to the wavelength. So for example a half wavelength electrode spacing would correspond to $\gamma=1 / 2$. From equation (23) at $x_{3}=h$ 


$$
\begin{aligned}
\sum_{r=1}^{8(2 N+1)(2 M+1)} A^{(r)} \phi^{q,(r)} e^{-\jmath k_{3}^{(r)} h} & =V_{0} \operatorname{sinc}\left(\left(k_{1}+G_{1}^{q}\right) \frac{p_{1}}{2}\right) \\
& \times \operatorname{sinc}\left(\left(k_{2}+G_{2}^{q}\right) \frac{p_{2}}{2}\right), q=1, \ldots(2 N+1)(2 M+1)
\end{aligned}
$$

and at $x_{3}=0$

$$
\sum_{r=1}^{8(2 N+1)(2 M+1)} A^{(r)} \phi^{q,(r)}=0 .
$$

Equations $(26),(27),(29)$ and $(30)$ constitute $8(2 N+1)(2 M+1)$ equations in the $8(2 N+$ $1)(2 M+1)$ unknowns $A^{(r)}$. Hence this system of linear equations can be solved and the displacements, stresses etc. can be examined using equation (23). One advantage of studying piezoelectric composites is that the electrical operating characteristics provide an alternative means of deriving the dispersion curves. The admittance $(Y)$ expresses the ease with which an alternating current flows through the transducer and the resonant modes are signified by maxima in the real part of the admittance. Using continuity of the electrical potential at the front interface [18] it can be shown that

$$
\begin{gathered}
Y\left(k_{1}, k_{2}, \omega\right)=\jmath \omega \\
\sum_{q=1}^{(2 N+1)(2 M+1)}\left[\sum_{r=1}^{8(2 N+1)(2 M+1)} A^{(r)}\left(D_{3}^{q,(r)}-\epsilon_{0}|\kappa| \phi^{q,(r)}\right) e^{-j k_{3}^{(r)} h}\right] \\
\times p_{1} \operatorname{sinc}\left(\left(k_{1}+G_{1}^{q}\right) \frac{p_{1}}{2}\right) p_{2} \operatorname{sinc}\left(\left(k_{2}+G_{2}^{q}\right) \frac{p_{2}}{2}\right)
\end{gathered}
$$

where $\kappa=\sqrt{\left(k_{1}+G_{1}^{q}\right)^{2}+\left(k_{2}+G_{2}^{q}\right)^{2}}$.

\section{Frequency dependent, viscoelastic loss model}

The degree of mechanical loss is usually expressed in terms of a dimensionless loss tangent $\tan \delta[21]$, a mechanical factor $Q$, or an attenuation coefficient $\alpha$ [22]. Frequency dependent loss can be introduced into the ceramic via $\tan \delta$ using

$$
\tan \delta=\left(\frac{\omega}{\omega_{0}}\right)\left(\frac{1}{Q_{0}}\right)
$$


where $\omega$ is the natural frequency, $Q_{0}$ is the measured mechanical $Q$ factor and $\omega_{0}$ is the natural frequency at which $Q_{0}$ is measured. Equation (32) is used to define the imaginary parts of the elastic constants in equations (7) and (11) using

$$
c_{i j k l}^{V^{p, q}}=c_{i j k l}^{V^{p, q}}(1+\jmath \tan \delta)
$$

The polymer phase is assumed to be isotropic and so the elastic constants are expressed in terms of the complex Lamé coefficients $\mu$ and $\lambda$, defined as

$$
\mu=\mu^{\prime}+\jmath \mu^{\prime \prime} \quad \text { and } \quad \lambda=\lambda^{\prime}+\jmath \lambda^{\prime \prime}
$$

so that the complex velocities are given by [23]

$$
v_{s}=\sqrt{\frac{\mu^{\prime}+\jmath \mu^{\prime \prime}}{\rho}} \quad, \quad v_{l}=\sqrt{\frac{\left(\lambda^{\prime}+2 \mu^{\prime}\right)+\jmath\left(\lambda^{\prime \prime}+2 \mu^{\prime \prime}\right)}{\rho}},
$$

where $v_{s}$ is the shear velocity, $v_{l}$ is the longitudinal velocity and $\rho$ is the density. The loss is however usually expressed for these materials in terms of a longitudinal attenuation coefficient $\left(\alpha_{l}\right)$ and a shear attenuation coefficient $\left(\alpha_{s}\right)$. Using the Voigt model for internal friction these can be related to $\tan \delta$ via [22]

$$
\tan \delta_{s}=\frac{2 \alpha_{s}\left|v_{s}\right|}{\omega} \quad, \quad \tan \delta_{l}=\frac{2 \alpha_{l}\left|v_{l}\right|}{\omega}
$$

The frequency dependency can be included via the attenuation coefficients using $\alpha_{l / s}=$ $\alpha_{l / s}^{0}\left(\omega / \omega_{0}\right)^{2}$, where $\alpha_{l / s}^{0}$ is the experimentally measured attenuation coefficient at frequency $\omega_{0}$. Using Equations (35) and (36) the imaginary parts of the Lamé coefficients are then given by

$$
\mu^{\prime \prime}=\sqrt{\frac{8 \alpha_{s}^{4} \mu^{\prime 4}}{\omega^{4} \rho^{4}}\left(1+\sqrt{1+\frac{\rho^{2} \omega^{4}}{4 \alpha_{s}^{4} \mu^{\prime 4}}}\right)}
$$

and

$$
\lambda^{\prime \prime}=\sqrt{\frac{8 \alpha_{l}^{4}\left(\lambda^{\prime}+2 \mu^{\prime}\right)^{4}}{\omega^{4} \rho^{4}}\left(1+\sqrt{1+\frac{\rho^{2} \omega^{4}}{4 \alpha_{l}^{4}\left(\lambda^{\prime}+2 \mu^{\prime}\right)^{4}}}\right)}-2 \mu^{\prime \prime} .
$$

So given $\alpha_{l / s}^{0}, \omega_{0}, \lambda^{\prime}, \mu^{\prime}, \rho$ and $\omega$, equations (37) and (38) can be used to give the complex valued elasticity tensor in equation (7) and associated Fourier coefficient in (11). The degree of shear loss in the polymer phase can be varied by multiplying the shear 
attenuation coefficient $\alpha_{s}$ by a parameter $\zeta$. The effect that changing from low shear attenuation to high shear attenuation has on the frequency band gap surrounding the fundamental thickness mode of the transducer will be investigated in Section 5 .

\subsection{Implementation}

From section 2.3 the vibrational modes of the transducer must satisfy the system of equations $X A^{(r)}=Q$, given by equations (26), (27), (29) and (30), where $X(k, \omega)$ is an $8(2 N+1)(2 M+1) \times 8(2 N+1)(2 M+1)$ matrix and $Q(k)$ is a column vector of length $8(2 N+1)(2 M+1)$. In the first instance the short circuit situation given by $V_{0}=0$ is examined, that is $Q=0$. The matrix $X$ is ill-conditioned and to help obviate this problem the matrix entries are balanced by scaling the parameters of the model (see Table 1). Each of the parameters is made $\mathrm{O}(1)$ by a judicious choice of the scalings $\alpha, \beta, \gamma$ and $\varphi$ so that five equations in four unknowns must be satisfied. This is done by scaling the thickness $h$ by specifying $\beta$, scaling the density $\rho$ by specifying $\alpha$, scaling the piezoelectric stress tensor $e_{i j k}$ by specifying $\varphi$, scaling the elasticity tensor $c_{i j k l}$ by specifying $\gamma$ and this results in an appropriate scaling for the permittivity tensor $\epsilon_{i j}$. Normally the modes are determined by adjusting the frequency $\omega$ and complex wavenumbers $k$ to make the determinant of $X$ equal to zero. As the number of Fourier coefficients are increased within the PWE method the dimensions of $X$ increase and the determinant becomes too large to be stored. This problem occurs due to the exponential terms which arise when calculating the boundary conditions at $x_{3}=h$. This term affects the rows between 1 and $3(2 N+1)(2 M+1)$ and $6(2 N+1)(2 M+1)+1$ and $7(2 N+1)(2 M+1)$ in the matrix $X$. To prevent the problem occurring these row entries are multiplied by a scale factor given by $e^{\operatorname{Max}_{r}\left\{k_{3}^{\prime \prime}(r)\right\} h}$, where $k_{3}^{\prime \prime(r)}$ is the imaginary part of the wavenumber $k_{3}^{(r)}$. There are also numerical instabilities as the determinant of $X$ approaches zero due to illconditioning, and Tikhonov regularisation [24] is used here to circumvent this. The matrix $X$ is converted to a real, symmetric form by multiplying it by its complex, conjugate $X^{*}$. The zero eigenvalues of $X$ are then translated along the real axis, away from the origin, by adding a small amount, $\mu$, to give 


$$
\left(X^{*} X+\mu I\right) A^{(r)}=X^{*} Q
$$

The determinant of $X^{*} X+\mu I$ is still large and so minima in the cost function surface $\log \left|X^{*} X+\mu I\right|$ are found, parameterised by the angular frequency, $\omega$, and complex wavenumbers $k_{1}$ and $k_{2}$.

Table 1:

Figure 2:

The effects of Tikhonov regularisation are shown for a 2-2 composite in Figure 2 for $N=3, M=0$. Ill-conditioning in the determinant calculation is indicated by the noise in plot (a) which corresponds to no regularisation being used. A major improvement can already be seen by making the matrix real and symmetric in plot (b). However the introduction of the small parameter $\mu$ results in the smooth curve in plot (c). Truncated Singular Value Decomposition [25] was also investigated but proved to be not as robust as Tikhonov regularisation in this instance. The algorithm for obtaining a particular mode for the 2-2 composite design sets $k_{2}$ equal to zero, $k_{1}$ to a real number (initially) and then searches the cost function surface in the frequency direction until a number of local minima are found. These interim minima are used as the initial values for a search in the direction of the imaginary part of $k_{1}$, although here the algorithm stops at the first local minimum. This orthogonal stepping procedure is then performed for a range of $k_{1}$ values.

\section{Comparison with finite element modelling and ex- perimental data}

The methodology presented in the previous sections is illustrated here by investigating the modal behaviour of some composite transducers. In this section the PWE method is compared with experimental measurements and FE results. 
Table 2:

Table 3:

Table 4:

Table 5:

Figure 3:

In Figure 3 the PWE method is compared to FE modelling [5] by examining the impedance characteristics of device A (see Table 2) with a range of polymer phase materials. The device has a half-wavelength electrode patterning on the top surface. Plot (a) shows the response when using a standard hardset polymer HY1300/CY1301 [5] (see Table 4). There is good agreement between both methods in the location of the modes (the thickness mode is around $0.5 \mathrm{MHz}$ ) but the PWE method overpredicts the magnitude of the impedance at the low frequency Lamb modes. The introduction of the high shear attenuation materials in plots (b) and (c) demonstrates that these unwanted low frequency Lamb modes can be damped down whilst maintaining a reasonable fundamental thickness mode response magnitude. The important point to note is that this enhancement of the device performance is predicted in a similar fashion by both modelling techniques. So although the PWE methods has difficulty here in the quantitative prediction of the impedance magnitude the modes, the qualitative improvements in performance are still evident.

Figure 4:

Figure 5:

Figure 6:

The PWE method is then compared to experimental and FE modelling data for device B in Table 2 with a HY1300/CY1301 hardset polymer phase [5]. A dispersion diagram showing the dependency of the phase velocity on the frequency is shown in Figure 4. In 
addition to predicting the Lamb modes the PWE method also predicts the presence of bulk waves and interpillar modes. These latter waves are excited by the spacing between adjacent pillars and diagonally opposite pillars, and are displayed as constant wavelength 'loadlines' in the dispersion diagram. As such the loadlines should appear as straight lines in this dispersion diagram. The slight deviations from linearity which occur in Figure 4 are indicative of the accuracy of the method in locating these modes. The deviation is about 5 per cent and this gives a rough measure of the accuracy of the method. There is good agreement between all three sets of results although the FE model predicts a larger frequency range for the $a_{0}$ mode than was observed experimentally. It should be borne in mind however that the PWE method is computationally less intensive than the FE model and its strength lies in providing a fast, qualitative prediction of the transducer's characteristics.

The number of Fourier coefficients used in the PWE method plays an analogous role to that of the spatial discretisation used in the FE analysis. The balance between computational cost and model accuracy can therefore be controlled in both methods. The accuracy can be gauged by comparison with experimental data and by examining the convergence of the model predictions as the computational complexity is increased. A systematic study of the convergence of the PWE method and its dependency on the number of Fourier coefficients has not yet been conducted. Comparing the computational cost of the two methods is difficult at present for the following reasons. The FE analysis conducted in this paper used a commercial code which has been highly optimised for computational efficiency [19] whereas the PWE method was implemented using a bespoke, suboptimal coding (in FORTRAN77). The FE analysis is for a transducer of finite lateral dimensions whereas the PWE method is for a periodic composite of infinite lateral extent. Thus reflections from the ends of the plate are included in the FE analysis and this explains some of the differences in this case. A quantitative comparison of the speed of each method is therefore problematic at present. However, it is clear from the simulations performed in this paper that the PWE method is at least an order of magnitude faster, although one must bear in mind the aforementioned distinctions in their implementations. 
The standard classification of the modes is problematic here as the supporting medium is heterogeneous, anisotropic, lossy and piezoelectric. As such the descriptions of the waves in terms of their symmetry, or as Lamb, Rayleigh, bulk waves etc. are only psuedodescriptions and the actual behaviour is far more complex. Identification of modes is aided by spatial and/or temporal plots of the displacement, the Poynting vector and the electrical potential. To illustrate the mode identification process, the $a_{0}$ mode is investigated. Figure 5 highlights the flexural nature of this mode, Figure 6 shows the elliptical motion of the internal dynamics (with the associated reversals in the direction of rotation) and demonstrates that the energy is predominantly at the plate boundaries. These are all characteristics of a flexural Lamb wave.

\section{Damping of unwanted lateral modes}

In this section, device $\mathrm{C}$ (see Table 2) with a standard hardset polymer (see Table 4) is investigated. To simulate the effect of increasing the shear attenuation a scaling parameter $\zeta$ is used to scale the shear attenuation coefficient, $\alpha_{s}^{0}$.

Figure 7:

Figure 7 shows two lossy dispersion diagrams (phase velocity versus complex wavenumber) which highlight the effect of introducing high shear loss into the passive phase. The plots are shaded according to the magnitude of the imaginary part of the wavenumber $\left(k_{1}\right)$, with the lighter shades corresponding to the highly attenuated modes. It can be seen that the degree of attenuation increases with frequency and eventually leads to each mode being cut off. By increasing the shear attenuation in the passive phase the frequency range of each mode is further reduced. As was seen in Figure 4 the experimental data displays mode cut-off and hence the inclusion of loss into the PWE method has considerably improved its predictive capabilities. Although not presented on these dispersion diagrams, it transpires that the extent of the band gap around the thickness mode widens as the shear attenuation in the polymer phase increases.

This can be visualised also by considering the electrical behaviour of the transducer. 
Figure 8:

The absolute value of the electrical conductance $(G)$ for this device is shown in Figure 8 , wherein the thickness mode can be identified by the central peak of the plot at around 0.65 MHz. The lower frequency maxima correspond to Lamb waves whilst the inter-pillar modes are the first set of peaks to the right of the thickness mode at around $1 \mathrm{MHz}$. Plotting the electrical conductance shows the relative importance of each mode and in this way it eradicates any spurious points found in the dispersion diagram. It can be seen that the higher shear attenuation passive phase (dashed line) damps out the unwanted inter-pillar modes. There also seems to be some slight increase in the magnitude of the $a_{0}$ Lamb mode. Note that high longitudinal attenuation in the passive phase is not investigated here since this will have the undesired effect of attenuating the energy in the thickness direction.

Figure 9:

Figure 10:

Figure 11:

To illustrate the mode identification process, two modes of vibration have been selected from the plot shown in Figure 8. In Figure 9 the displacement $u_{1}$ is negligible compared to $u_{3}$, which has its largest values at the faces of the transducer, within the ceramic. Of course this is at one instant in time and it is useful to examine the temporal evolution of various spatially fixed reference points within the device. Figure 10 shows that the ceramic pillars are moving vertically with very little motion in the $x_{1}$ direction (note the axes scales), however the polymer is being pulled sideways with no motion in the $x_{3}$ direction. The in-plane Poynting vector can be viewed by proportionally displacing the $x_{1}$ and $x_{3}$ components to show where the energy is stored. Figure 11 shows that the energy is distributed throughout the transducer, primarily in the thickness direction. The symmetrical displacement profile in both directions, the large amplitude of oscillation and 
the dominant displacement being in the $x_{3}$ direction all point to this being the thickness mode.

Figure 12:

Figure 13:

In Figure 12, a comparison between low and high shear attenuation in the passive phase is shown using the displacement at one instant in time for device C. The particular mode shown is that of the unwanted inter-pillar wave. It can clearly be seen in plot (b) that the introduction of the high shear loss damps out the vibrations in the passive phase seen in plot (a). To aid in the identification of this mode the spatial variation in the magnitude of the Poynting vector, integrated with respect to time over one period of oscillation, is shown in Figure 13. It can be seen that the energy is concentrated in the polymer phase which is characteristic of an inter-pillar mode.

\section{Conclusions}

The plane wave expansion (PWE) method is a frequency domain approach for studying the modal behaviour of periodic piezoelectric composite transducers. It is shown in this paper that the method can be extended to incorporate frequency dependent loss in both phases. One advantage of this approach over time domain methods is that information on low amplitude or high frequency modes is retained. A strict comparison between the PWE and FE methods in terms of computational cost and accuracy is difficult at present but it would appear that the PWE method provides a more qualitative prediction at a fraction of the computational time. One advantage of investigating piezoelectric composites is that the electrical characteristics provide an additional means of inferring the mechanical wave dispersion properties. This approach can complement the harmonic analysis which relies on searching the frequency/complex wavenumber parameter space for zeros of the determinant of a large, ill-conditioned matrix. This paper has also shown 
however that the use of scaling and Tikhonov regularisation greatly improves the conditioning of this latter approach. Although the standard classification of the modes is difficult, as the supporting medium is heterogeneous, anisotropic, lossy and piezoelectric, pseudo-descriptions of the main supported modes of vibrations can be obtained by using spatial and/or temporal plots of the displacement and the Poynting vector. It is shown that there is good agreement between the PWE method, FE analysis and experimental data. Dispersion characteristics for low and high shear attenuation in the passive phase were also compared and this showed that the use of a high shear loss polymer as the passive phase in a 2-2 composite transducer results in an improved stop bad gap around the fundamental thickness mode.

\section{References}

[1] C.J. Harvey, J.M. Pilcher, R.J. Eckersley, M.J.K. Blomley and D.O. Cosgrove, "Advances in Ultrasound", Clin. Rad., vol. 57, no. 3, pp 157-177, Mar. 2002.

[2] K.C. Benjamin, "Recent Advances in 1-3 Piezoelectric Polymer Composite Transducer Technology for AUV/UUV Acoustic Imaging Applications", J. Electroceramics, vol. 8, no. 2, pp. 145-154, Aug. 2002.

[3] M. Moles, N. Dube, S. Labbe and E. Ginzel, "Review of Ultrasonic Phased Arrays for Pressure Vessel and Pipeline Weld Inspections", J. Pressure Vessel Technology-Trans. ASME, vol. 127, no. 3, pp. 351-356, Aug. 2005.

[4] G. Hayward and J. Hyslop, "Determination of Lamb Wave Dispersion in Data in Lossy Anisotropic Plates Using Time Domain Finite Element Analysis. Part 1: Theory and Experimental Verification", IEEE Trans. UFFC., vol. 53, no. 2, pp. 443-448, Feb. 2006.

[5] G. Hayward and J. Hyslop, "Determination of Lamb Wave Dispersion in Data in Lossy Anisotropic Plates Using Time Domain Finite Element Analysis. Part 2: Application 
to 2-2 and 1-3 Piezoelectric Composite Transducer Arrays", IEEE Trans. UFFC., vol. 53, no. 2, pp. 449-455, Feb. 2006.

[6] H.P. Savakus, K.A. Klicker and R.E. Newnham, "PZT-Epoxy Piezoelectric Transducer: A Simplified Fabrication Procedure", Mat. Res. Bull., vol. 16, pp. 677-680, 1981.

[7] T.R. Gururaja, W.A. Schulze, L.E. Cross, R.E. Newnham, B.A. Auld and Y.J. Wang, "Piezoelectric composite materials for ultrasonic transducer applications. Part 1: Resonant modes of vibration of PZT rod-polymer composites", IEEE Trans. Sonics and Ultrasonics, vol. SU-22, no. 4, pp. 481-498, July 1985.

[8] T.R. Gururaja, W.A. Schulze, L.E. Cross and R.E. Newnham, "Piezoelectric composite materials for ultrasonic transducer applications. Part 2: Evaluation of ultrasonic medical applications", IEEE Trans. Sonics and Ultrasonics, vol. SU-22, no. 4, pp. 499-513, July 1985.

[9] Y. Shui, X. Geng and Q.M. Zhang, "Theoretical Modelling of Resonant Modes of Composite Ultrasonic Transducers", IEEE Trans. UFFC., vol. 42, no. 4, pp. 766-773, July 1995.

[10] X. Geng and Q.M. Zhang, "Evaluation of Piezocomposites for Ultrasonic Transducer Applications - Influence of the Unit Cell Dimensions and the Properties of Constituents on the Performance of 2-2 Piezocomposites", IEEE Trans. UFFC., vol. 44, no. 4, pp. 857-872, July 1997.

[11] D. Certon, F. Patat, F. Levassort, G. Feuillard and B. Karlsson, "Lateral Resonances in 1-3 Piezoelectric Periodic Composites: Modelling and Experimental Results", JASA, vol. 101, no. 4, pp. 2043-2051, Apr. 1997.

[12] J.O. Vasseur, B. Djafari-Rouhani, L. Dobrzynski, M.S. Kushwaha and P. Halevi, "Complete acoustic band gaps in periodic fibre reinforced composite materials: The carbon/epoxy composite and some metallic systems", J. Phys.: Condens. Matter, vol. 6, pp. 8759-8770, 1994. 
[13] D. Certon, O. Casula, F. Patat and D. Royer, "Lateral Resonances in 1-3 Piezoelectric Periodic Composites: Modelling and Experimental Results", JASA, vol. 101, no. 4, pp. 2043-2051, Apr. 1997.

[14] G. Hayward and J.A. Hossack, "Unidimensional Modelling of 1-3 Composite Transducers", JASA, vol. 88, no. 2, pp. 599-607, 1990.

[15] S. Ballandras, M. Wilm, P. Edoa and A. Soufyane, "Finite-Element Analysis of Periodic Piezoelectric Transducers", JASA, vol. 93, no. 1, pp. 702-711, 2003.

[16] ANSYS Europe Ltd, 1st Floor, Waterloo House, Riseley Business Park, Riseley, Berkshire, RG7 1NW, United Kingdom, www.ansys.com.

[17] A.C.S. Parr, A. Troge, R.L. O'Leary, R.A. Pethrick and G. Hayward, "Performance of Periodic Piezoelectric Composite Arrays Incorporating a Passive Phase Exhibiting Anisotropic Properties" Proceedings of the 2005 IEEE International Ultrasonics Symposium, Rotterdam, 18-21 Sept, pp1073-1076, 2005.

[18] M. Wilm, S. Ballandras, V. Laude, and T. Pastureaud, "A Full 3D Plane-WaveExpansion Model for 1-3 Piezoelectric Composite Structures", JASA, vol. 112, no. 3, pp. 943-952, Sept. 2002.

[19] PZFlex: Distributed by Weidlinger Associates, 4410 El Camino Real, Suite 110, Los Altos, CA, 94022, U.S.A, www.wai.com.

[20] "ANSI/IEEE Standard on Piezoelectricity", IEEE Trans. UFFC., vol. 43, no. 5, pp. 717-771, Sept. 1996.

[21] N.G. McCrum, B.E. Read and G. Williams, Anelastic and Dielectric Effects in Polymeric Solids, John Wiley and Sons, 1967.

[22] R.S. Lakes, Viscoelastic Solids, CRC Press, London, 1999.

[23] M.J.S. Lowe, "Matrix Techniques for Modeling Ultrasonic Waves in Multilayered Media", IEEE Trans. UFFC., vol. 42, no. 4, pp. 525-542, Jul. 1995. 
[24] A. Neumaier, "Solving Ill-conditioned and Singular Linear Systems; A Tutorial on Regularization", IEEE Trans. UFFC., vol. 44, no. 5, pp. 1018-1026, Sept. 1998.

[25] G.R. Liu and X. Han, Computational Inverse Techniques in Nondestructive Evaluation, CRC Press, London, 2003.

[26] R.L. O'Leary, G. Smillie, G. Hayward and A.C.S. Parr, CUE Materials Database, Centre for Ultrasonic Engineering, University of Strathclyde, Glasgow, Scotland, June 2002, www.cue.ac.uk.

[27] Ferroperm UK Ltd, Vauxhall Industrial Estate, Ruabon, Wrexham, United Kingdom, LL14 6HA, www.ferroperm.co.uk.

[28] Huntsman Advanced Materials Ltd, Ickleton Road, Duxford, Cambridge, United Kingdom, CB2 4QA, www.huntsman.com. 